\title{
Magnetic resonance imaging findings within the posterior and lateral columns of the spinal cord extended from the medulla oblongata to the thoracic spine in a woman with subacute combined degeneration without hematologic disorders: a case report and review of the literature
}

Samira Rabhi ${ }^{{ }^{*}}$, Mustapha Maaroufi ${ }^{2}$, Hajar Khibri ${ }^{1}$, Faouzy Belahsen ${ }^{3}$, Siham Tizniti ${ }^{2}$, Rhizlane Berrady ${ }^{1}$ and Wafaa Bono ${ }^{1}$

\begin{abstract}
Introduction: Subacute combined degeneration of the spinal cord is a rare cause of demyelination of the dorsal and lateral columns of the spinal cord and is a neurological complication of vitamin $B_{12}$ deficiency. Subacute combined degeneration without anemia or macrocytosis is rare.

Case presentation: We present a case of cobalamin deficiency in a 29-year-old Moroccan woman who presented with subacute combined degeneration without evidence of anemia or macrocytosis. Magnetic resonance imaging of the spinal cord demonstrated abnormal hyperintense signal changes on T2-weighted imaging of the posterior and lateral columns from the medulla oblongata to the thoracic spine. A diagnosis of subacute combined degeneration of the spinal cord was considered and confirmed by low serum cobalamin. The patient was treated with vitamin $B_{12}$ supplements and showed improvement in her clinical symptoms.
\end{abstract}

Conclusion: Physicians should diagnose subacute combined degeneration in patients early by having a high index of suspicion and using diagnostic tools such as magnetic resonance imaging.

\section{Introduction}

Vitamin $B_{12}$ deficiency usually presents with various hematological, gastrointestinal and neuropsychiatric manifestations. Commonly seen neuropsychiatric manifestations include myelopathy, neuropathy, dementia, neuropsychiatric abnormalities and, rarely, optic nerve atrophy. Subacute combined degeneration (SCD) of the spinal cord is an uncommon cause of myelopathy but is the most frequent clinical manifestation of vitamin $\mathrm{B}_{12}$ deficiency [1]. As anemia is a common early symptom

\footnotetext{
* Correspondence: rabhisamira@gmail.com

'Department of Internal Medicine, Hassan II University Hospital, Fez, Morocco Full list of author information is available at the end of the article
}

leading to the diagnosis of vitamin $\mathrm{B}_{12}$ deficiency, neurological symptoms have often been considered to be late manifestations and typically occur after the development of anemia [2]. We present the magnetic resonance imaging (MRI) scans of a patient with SCD involving the lateral and posterior columns extended to segments of spinal cord and without anemia or macrocytosis.

\section{Case presentation}

A 29-year-old Moroccan woman came to our institution complaining of numbness and tingling of four months' duration in both lower limbs, with unsteady gait and easy falling and urine incontinence. The patient's 
background and history did not reveal preexisting diabetes mellitus, alcohol addiction, vegetarian food preference or gastrointestinal symptoms. She did not mention any fever, night sweats or itching. On physical examination, her temperature was $37.4^{\circ} \mathrm{C}$, her pulse was 80 beats/minute and her blood pressure was 120/83 $\mathrm{mmHg}$. She had no pallor or icterus and no lymphadenopathy, edema, splenomegaly or hepatomegaly. On neurological examination, her deep tendon reflexes were hyperactive in the upper and lower extremities. Babinski's sign, Romberg's sign and Lhermitte's sign were present. Vibration and joint position sense examination were evaluated as decreased. However, there was no decrease in light touch sensation. Her laboratory examination values were unremarkable: white blood cell count $8,500 / \mathrm{mm}^{3}$, hemoglobin $13 \mathrm{~g} / \mathrm{dL}$, mean corpuscular volume $97 / \mu^{3}$, platelets $225,000 / \mathrm{mm}^{3}$ and thyroid-stimulating hormone $1.5 \mathrm{mU} / \mathrm{L}$.

The initial MRI examination of the cervical and dorsal spine was performed using a 1.5-T unit and showed an area of hyperintensity involving the dorsal and lateral columns from the medulla oblongata (Figure 1) to the thoracic spine (Figure 2, Figure 3 and Figure 4) on T2weighted images. This area was not enhanced after the addition of gadolinium. The axial images revealed involvement of the posterior and lateral columns bilaterally (Figures 4 and 5), which was highly suggestive of SCD. The serum vitamin $B_{12}$ level was collapsed to $25 \mathrm{pg} / \mathrm{mL}$ (normal range, 180 to $914 \mathrm{pg} / \mathrm{mL}$ ), and her serum vitamin E level was normal.

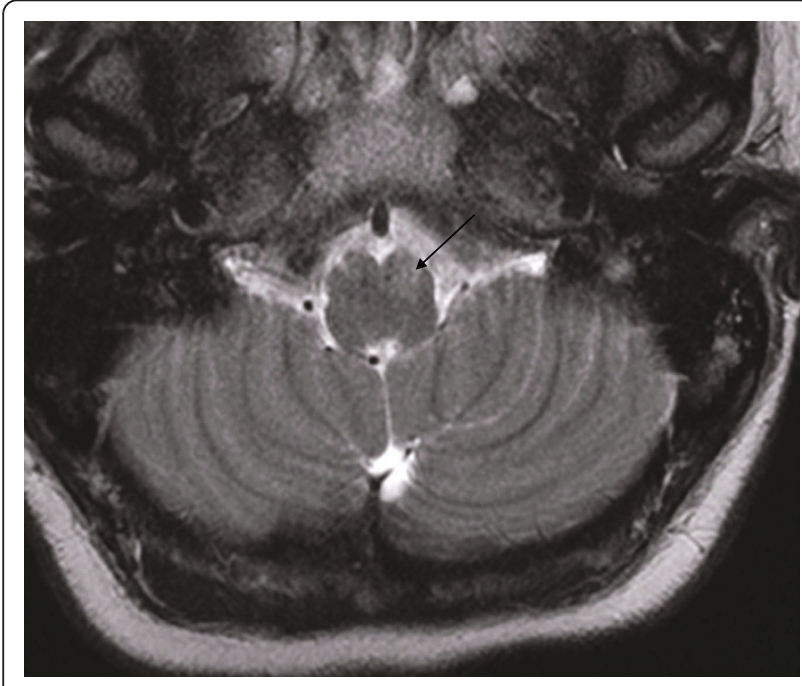

Figure 1 Transverse T2-weighted magnetic resonance imaging (MRI) scan of the posterior cerebral fossa showing symmetric signal intensity within the medulla oblongata before treatment

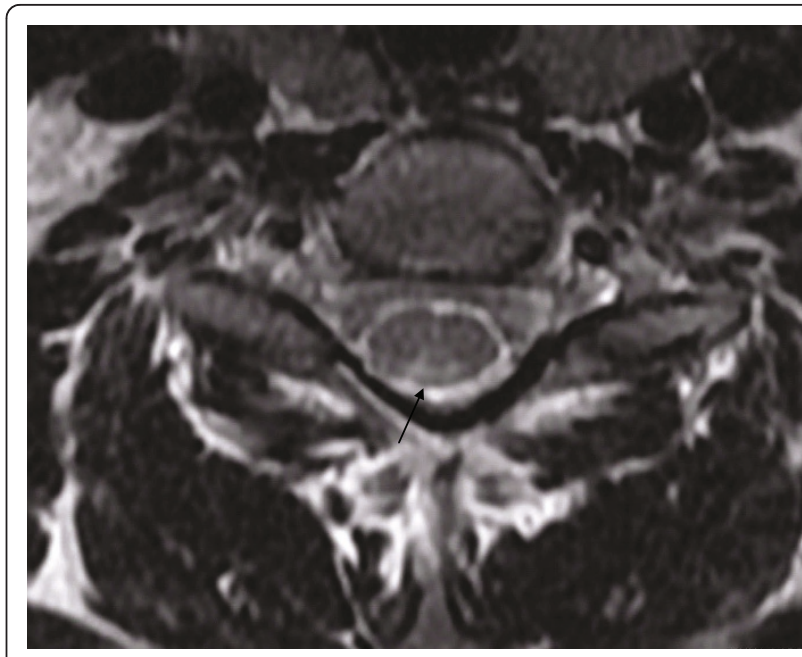

Figure 2 Transverse T2-weighted MRI scan of the cervical spinal cord at the C2 level demonstrating bilateral symmetric signal intensity within the dorsal columns (inverted V sign) before treatment.

Bone marrow aspirates showed a medullary megaloblastosis. A Schilling test was not available. Upper gastrointestinal examination revealed fundic atrophic gastritis. Parietal cell antibodies and anti-intrinsic factor were positive. The neurological symptoms totally disappeared two months after intramuscular supplementation of vitamin $B_{12} 1,000 \mu \mathrm{g}$ daily for one week, then weekly for two weeks, and then monthly. The MRI scan abnormalities were significantly improved (Figure 6).

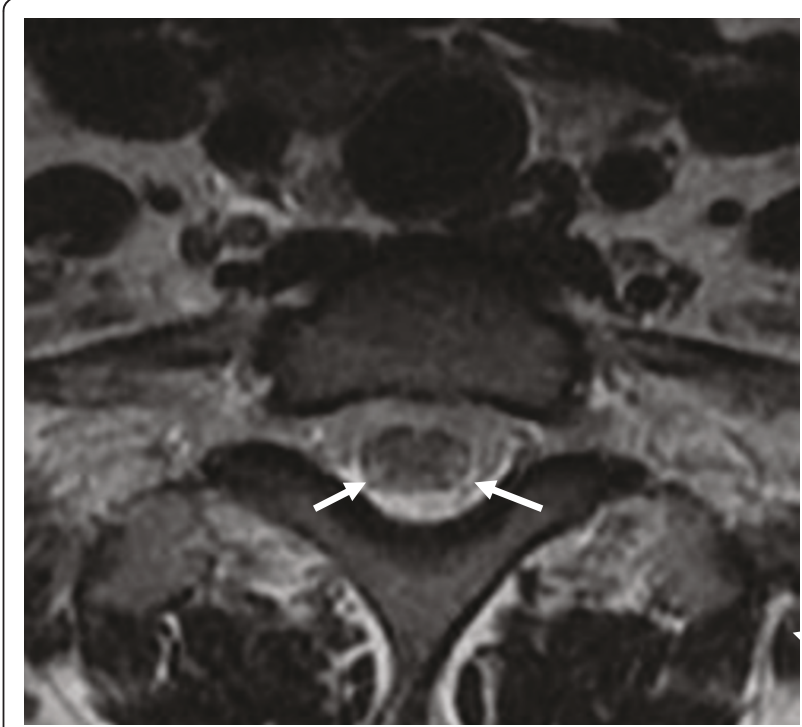

Figure 3 Transverse T2-weighted MRI scan of the cervical spinal cord at the $\mathrm{C} 7$ level demonstrating symmetric signal intensity within the lateral and dorsal columns before treatment 


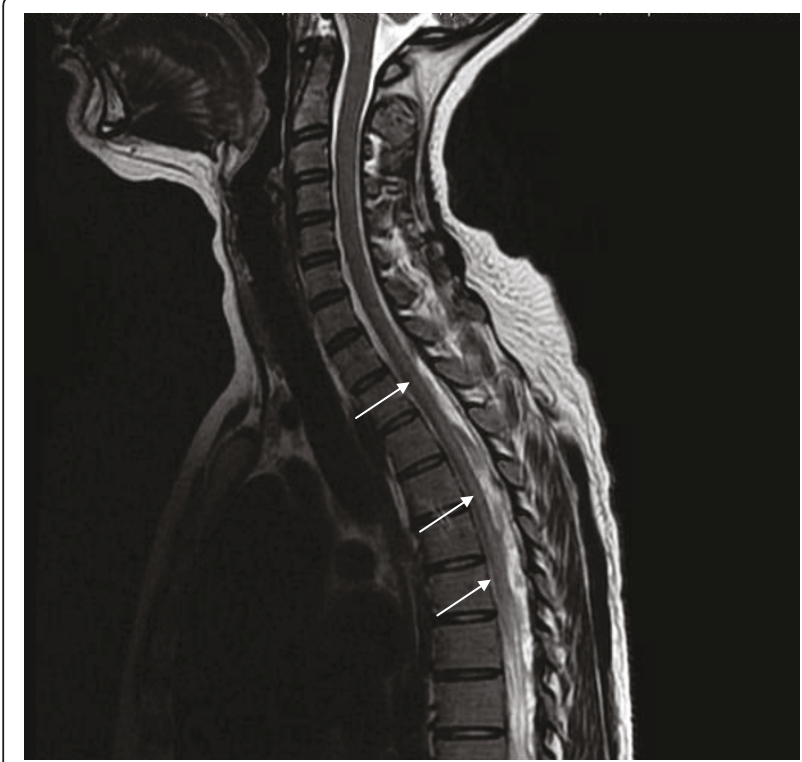

Figure 4 Sagittal T2-weighted MRI scan showing the dorsal spinal cord with hyperintensity involving the posterior and lateral columns before treatment.

\section{Discussion}

We describe new MRI findings of the spinal cord in $S C D$ revealing vitamin $B_{12}$ deficiency without anemia and or macrocytosis. The high-signal intensity, T2weighted MRI findings within the posterior and lateral columns extended from the medulla oblongata to the thoracic spine.

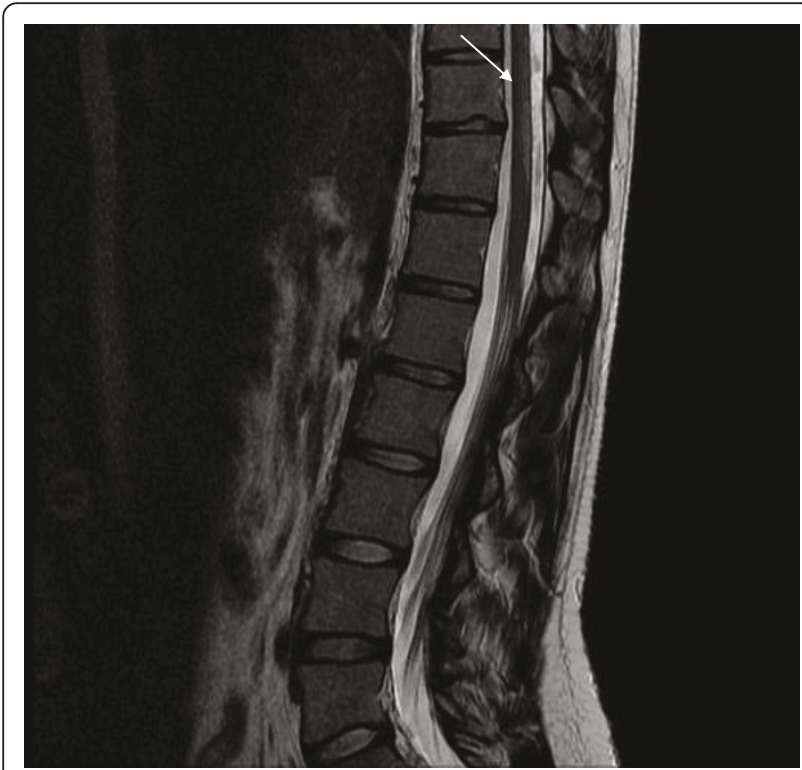

Figure 5 Sagittal T2-weighted MRI scan showing an area of hyperintensity involving the bilateral posterior and lateral columns of the thoracic and lumbar junction before treatment.

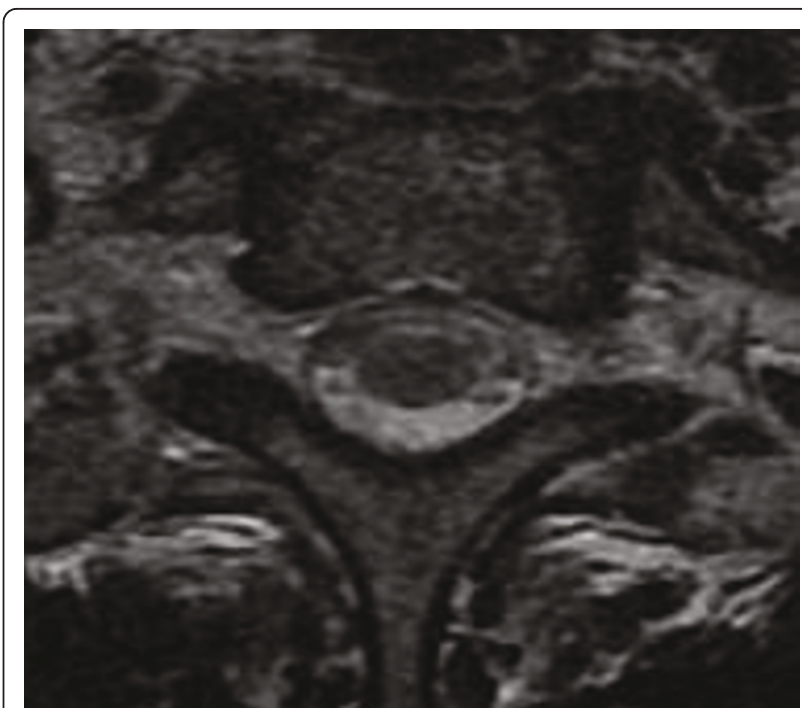

Figure 6 Transverse T2-weighted MRI scan of the cervical spinal cord at the C7 level with normal signal intensity of the lateral and dorsal columns after treatment.

The myelopathy of vitamin $B_{12}$ deficiency (or SCD) is characterized neuropathologically by degeneration of myelin and axonal loss [3]. It is clear now that the neuropathological lesions in SCD are due to overproduction of myelinolytic tumor necrosis factor $\alpha$ (TNF- $\alpha)$ and to the reduced synthesis of the two neurotrophic agents epidermal growth factor (EGF) and interleukin-6. This deregulation of the balance between TNF- $\alpha$ and EGF synthesis is induced by cobalamin deficiency [4].

Neuropathological studies show the main lesions to be in the posterior and lateral columns, predominantly in the upper thoracic and midthoracic regions [5]. The MRI findings of the spinal cord in SCD are high-signal intensity on T2-weighted images within the posterior or lateral columns. Brain lesions of vitamin $\mathrm{B}_{12}$ deficiency over the medulla oblongata, pons, mesencephalon and crus cerebelli have also been reported [6]. SCD can also result from common variable immunodeficiency syndrome, paraneoplastic malabsorption, folate deficiency, acute monoblastic leukemia and nitrous oxide anesthesia [7].

The main symptoms of SCD are paresthesia, stiffness, numbness or tingling of the limbs; sensory ataxia; and impaired vibration and joint position sensation. Spastic paraparesis may develop if SCD is left untreated. Babinski's sign may be present, and the deep tendon reflexes are variable [8]. If these symptoms are associated with macrocytic anemia, the possibility of SCD should be strongly considered. Usually, vitamin $\mathrm{B}_{12}$ deficiency is detected on the basis of hematological abnormalities such as macrocytic megaloblastic anemia or macrocytosis, but it was noted to be associated with only 
neuropsychiatric abnormalities in $28 \%$ of one population studied [2]. The hematologic abnormalities of vitamin $\mathrm{B}_{12}$ deficiency (macrocytic anemia) may develop after neurologic abnormalities. Some patients with SCD might have minimal symptoms without hematologic abnormalities initially, such as acroparesthesia and Lhermitte's sign only. At this moment, in the early stage, in addition to blood vitamin $B_{12}$ and homocysteine levels, spinal MRI may be a good diagnostic tool [9]. Once the diagnosis of SCD is suspected, treatment with vitamin $B_{12}$ injection should be started as early as possible to avoid irreversible neurologic damage. Improvement in myelopathy may occur if vitamin $B_{12}$ therapy is started early in the course of the disease. The resolution of the MRI changes in our case correlated well with the clinical improvement $[8,10]$.

\section{Conclusion}

We report a case of an adult with SCD with new MRI findings from the medulla oblongata to the thoracic spine with high signal intensity on T2-weighted images within the posterior or lateral columns and without hematologic disorders. There have been few cases reported in the literature with extended lesions over several segments of the spinal cord. Complete recovery from the disease was not observed in the previous reports. Patients with SCD should be diagnosed early by their treating physicians having a high index of suspicion and using diagnostic tools such as MRI.

\section{Consent}

Written, informed consent was obtained from the patient for publication of this case report and accompanying images. A copy of the written consent is available for review by the Editor-in-Chief of this journal.

\section{Author details \\ 'Department of Internal Medicine, Hassan II University Hospital, Fez, Morocco. ${ }^{2}$ Department of Radiology, Hassan II University Hospital, Fez, Morocco. ${ }^{3}$ Department of Neurology, Hassan II University Hospital, Fez, Morocco.}

\section{Authors' contributions}

SR wrote the manuscript with comments and revision. MM prepared the figures. All authors read and approved the final manuscript.

\section{Competing interests}

The authors declare that they have no competing interests.

Received: 7 July 2010 Accepted: 27 April 2011 Published: 27 April 2011

\section{References}

1. Lee GR: Pernicious anemia and other causes of vitamin $B_{12}$ (cobalamin) deficiency. In Wintrobe's Clinical Hematology.. 10 edition. Edited by: Lee GR, Foerster J, Lukens J, Paraskevas F, Greer JP, Rodgers GM. Baltimore: Lippincott Williams 1999:941-964.

2. Lindenbaum J, Healton EB, Savage DG, Brust JC, Garrett TJ, Podell ER, Marcell PD, Stabler SP, Allen RH: Neuropsychiatric disorders caused by cobalamin deficiency in the absence of anemia or macrocytosis. $\mathrm{N} \mathrm{Eng/} \mathrm{J}$ Med 1988, 318:1720-1728.

3. Karantanas AH, Markonis A, Bisbiyiannis G: Subacute combined degeneration of the spinal cord with involvement of the anterior columns: a new MRI finding. Neuroradiology 2000, 42:115-117.

4. Scalabrino G, Carpo M, Bamonti F, Pizzinelli S, D'Avino C, Bresolin N, Meucci G, Martinelli V, Comi GC, Peracchi M: High tumor necrosis factor-a levels in cerebrospinal fluid of cobalamin-deficient patients. Ann Neurol 2004, 56:886-890.

5. Timms SR, Curé JK, Kurent JE: Subacute combined degeneration of the spinal cord: MR findings. AJNR Am J Neuroradiol 1993, 14:1224-1227.

6. Lee WJ, Hsu HY, Wang PY: Reversible myelopathy on magnetic resonance imaging due to cobalamin deficiency. J Chin Med Assoc 2008, 71:368-372.

7. Bou-Haidar P, Peduto AJ, Karunaratne N: Differential diagnosis of T2 hyperintense spinal cord lesions: part B. J Med Imaging Radiat Oncol 2009, 53:152-159.

8. Yamada K, Shrier DA, Tanaka H, Numaguchi Y: A case of subacute combined degeneration: MRI findings. Neuroradiology 1998, 40:398-400.

9. Fritschi J, Sturzenegger M: Spinal MRI supporting myelopathic origin of early symptoms in unsuspected cobalamin deficiency. Eur Neurol 2003, 49:146-150.

10. Katsaros VK, Glocker FX, Hemmer B, Schumacher M: MRI of spinal cord and brain lesions in subacute combined degeneration. Neuroradiology 1998, 40:716-719.

\section{doi:10.1186/1752-1947-5-166}

Cite this article as: Rabhi et al:: Magnetic resonance imaging findings within the posterior and lateral columns of the spinal cord extended from the medulla oblongata to the thoracic spine in a woman with subacute combined degeneration without hematologic disorders: a case report and review of the literature. Journal of Medical Case Reports 2011 5:166.

\section{Submit your next manuscript to BioMed Central and take full advantage of:}

- Convenient online submission

- Thorough peer review

- No space constraints or color figure charges

- Immediate publication on acceptance

- Inclusion in PubMed, CAS, Scopus and Google Scholar

- Research which is freely available for redistribution 